\title{
Social and Physical Anhedonia and Valence and Arousal Aspects of Emotional Experience
}

\author{
John G. Kerns, Anna R. Docherty, and Elizabeth A. Martin \\ University of Missouri
}

\begin{abstract}
Two studies examined whether self-reported anhedonia is associated with 2 facets of emotional experience, valence and arousal. In Study 1, in multiple assessments of emotional experience (e.g., naturalistic and lab contexts and social and nonsocial situations), people with elevated social anhedonia $(n=40)$ reported less intensity of positive affect than both controls $(n=30)$ and people with elevated perceptual aberration-magical ideation $(n=29)$. Social anhedonia was also associated with providing less emotional content when describing what it is like to experience positive situations. In contrast, both social anhedonia and perceptual aberration-magical ideation were associated with increased frequency of negative affect for their daily experiences. Moreover, social anhedonia was not associated with a decrease specifically in high-arousal emotions. In Study $2(n=339)$, social and physical anhedonia (but not perceptual aberration-magical ideation) were again associated with decreased self-reported positive affect to lab stimuli. In these studies, results were not statistically accounted for by personality, current mood, or current distress. Overall, results suggest anhedonia may be associated with a general decrease in self-reported positive affect intensity.
\end{abstract}

Keywords: anhedonia, emotion experience, valence, arousal, positive affect

Negative symptoms, such as anhedonia, are an important predictor of poor outcome in schizophrenia and are an increasingly important target for clinical interventions (Kirkpatrick, Fenton, Carpenter, \& Marder, 2006). Anhedonia refers to a loss of selfreported pleasure (Meehl, 1962), including for both social and physical experiences (L. J. Chapman, Chapman, \& Kwapil, 1995), and it is a prominent symptom in people with schizophrenia (e.g., Horan, Green, Kring, \& Nuechterlein, 2006). Social anhedonia also predicts future schizophrenia-spectrum disorders (Gooding, Tallent, \& Matts, 2005; Kwapil, 1998). Furthermore, there is evidence that negative symptoms like anhedonia can appear in the prodromal phase even before the emergence of psychotic symptoms (Hafner \& an der Heiden, 2003). Hence, understanding the nature of anhedonia might provide evidence about the nature of the liability for schizophrenia (Lenzenweger, 1999). Previous research has suggested a possible diminution of emotional experience in anhedonia (e.g., Kerns, 2006). In the current research, we examined whether anhedonia in people at risk for schizophreniaspectrum disorders was associated with a decrease in two specific facets of emotional experience, valence and arousal. In addition, we examined two possible ways in which emotional experience could be altered in anhedonia: (a) a generalized change in emotion or (b) a change in the types of emotional situations typically experienced by people with anhedonia.

John G. Kerns, Anna R. Docherty, and Elizabeth A. Martin, Department of Psychological Sciences, University of Missouri.

Work on this article was supported by National Institute of Mental Health Grant MH072706, National Institute on Drug Abuse Grant DA022405, and a MU Research Board grant.

Correspondence concerning this article should be addressed to John G. Kerns, Department of Psychological Sciences, University of Missouri, 214 McAlester Hall, Columbia, MO 65211. E-mail: KernsJ@missouri.edu
Although there is no agreed-on definition of emotion, emotions are often thought to be complex reactions to personally significant events that include feelings as well as physiological and behavioral changes (VandenBos, 2006). Emotion research involves many important questions, such as what causes an emotion, what are its neurobiological correlates, and what are its consequences for information processing and behavior (LeDoux, 2000; Phelps, 2006). We specifically examined emotional experience and whether anhedonia is associated with changes in the subjective experience of emotion. According to a recent review of research and theoretical views of emotional experience (Barrett, Mesquita, Ochsner, \& Gross, 2007), emotional experience involves multiple features. One possible core feature of emotional experience is valence, or a sense of pleasure or displeasure. Another very common feature of emotional experience is arousal, or a sense of activation or deactivation (e.g., "excited" is a high-arousal emotion, and "serene" is a low-arousal emotion). At the same time, it is thought that these features of emotional experience might interact with both the amount of attention given to affective feelings and the depth of conceptual knowledge used to process these feelings (Barrett et al., 2007; Frijda \& Sundararajan, 2007; Lambie \& Marcel, 2002). Therefore, emotional experience is thought to involve certain core affective features, such as valence and arousal, and how these affective features are experienced is thought to depend on attention and conceptual processing. The current study specifically focused on whether anhedonia is associated with changes in self-reported valence or arousal.

Some previous research has suggested that anhedonia might be associated with changes in the experience of valence and arousal. For valence, some previous research has suggested that anhedonia might be associated with decreased self-reported positive affect. For instance, anhedonia has typically been measured using trait self-report measures largely assessing whether people tend to find 
particular experiences pleasurable (e.g., L. J. Chapman, Chapman, \& Raulin, 1976). Moreover, anhedonia has been associated with decreased trait positive affect (Gooding, Davidson, Putnam, \& Tallent, 2002; including in people with schizophrenia, Horan \& Blanchard, 2003) and with decreased extraversion (Kerns, 2006; Mason, 1995; Ross, Lutz, \& Bailey, 2002; with one view of the nature of extraversion as largely reflecting trait levels of positive affect, Lucas \& Diener, 2001; Watson, Gamez, \& Simms, 2005). In contrast, anhedonia has been only weakly associated with neuroticism (Kerns, 2006; Mason, 1995; Ross et al., 2002). One view of neuroticism is that it largely reflects trait levels of negative affect (Watson et al., 2005), suggesting that anhedonia might be weakly associated with increased negative affect. Hence, some previous anhedonia research has suggested that it might be related to a reduction in positive affect and possibly to a small increase in negative affect. However, although anhedonia has been associated with trait measures of affect, some previous emotion research has found some dissociations between people's beliefs about how they typically feel as assessed by trait measures versus how they report feeling in current situations (Robinson \& Clore, 2002). Hence, we examined whether people with anhedonia would also report changes in emotional experience for specific situations.

In addition to possible changes in the experience of valence, some previous research has suggested that anhedonia could be related to changes in the experience of emotional arousal (i.e., sense of activation or deactivation). For instance, it has been found that anhedonia is associated with an atypical left hemisphere bias on the chimeric faces task (Luh \& Gooding, 1999). The typical right hemisphere bias on this task is thought to reflect right parietal functioning, with the right parietal area being associated with emotional arousal (Heller, 1994). At the same time, anhedonia has been associated with decreased scores on a questionnaire assessing trait affect intensity (Kerns, 2006). It is possible that a decrease in trait affect intensity could reflect a decrease in the experience of high-arousal emotions (for both positive emotions, e.g., excited and alert, and negative emotions, e.g., stressed and nervous; Barrett \& Russell, 1999). Therefore, it is possible that anhedonia might reflect a decreased experience of high-arousal emotions; however, to our knowledge this has not been examined in previous research.

On the basis of previous research, we examined whether anhedonia was associated with a decrease in positive affect, a small increase in negative affect, and a decrease in high-arousal emotions. In addition, in the current research we also examined the nature of any change of emotional experience in anhedonia. Although there are many possible ways in which emotional experience could be altered, the current research focused on two general possibilities for how emotional experience might be changed in anhedonia. One possibility is that people with anhedonia report a generalized change in emotional experience. The second possibility is that people with anhedonia differ from other people in the types of emotion-eliciting situations they tend to experience. These two possibilities make different predictions regarding when reports of emotion experience should be altered for people with anhedonia. If people with anhedonia have a generalized change in emotional experience, then one would expected to find it across situations and reporting formats. In contrast, if people with anhedonia differ in emotional experience because of the types of situations they tend to experience, then reports of their emotional experience should vary by the type of situation and also possibly by the type of reporting format.

In the current research, we examined five methodological factors that might reveal the specific types of situations and reporting formats in which people with anhedonia might selectively report altered emotion experience. One methodological factor is whether emotions concern reactions to daily life situations or to lab stimuli. If people with anhedonia report decreased emotion experience because they are less likely to experience emotion-eliciting events, then they should report decreased emotion for the idiosyncratic real-world situations they experience, but they should not report decreased emotion for lab stimuli because with lab stimuli every participant responds to the exact same stimulus. Evidence for this type of dissociation between real-world versus lab stimuli has been found in people with schizophrenia. Although people with schizophrenia report decreased positive affect (and increased negative affect) in their daily lives (e.g., Myin-Germeys, Delespaul, \& deVries, 2000), accumulating evidence suggests that people with schizophrenia do not report decreased positive affect for lab stimuli (Burbridge \& Barch, 2007; Horan et al., 2006; Kring, 1999). Previous research in people at possible risk for schizophrenia examining whether physical anhedonia is associated with a reduction in positive affect for emotional stimuli in lab situations has been very mixed (Berenbaum, Snowhite, \& Oltmanns, 1987; Ferguson \& Katkin, 1996; Fiorito \& Simons, 1994; Fitzgibbons \& Simons, 1992; Germans \& Kring, 2000), although to our knowledge this has not been examined in social anhedonia.

A second methodological factor is whether emotions concern social versus nonsocial situations. For example, people with social anhedonia report not enjoying social situations (Eckblad, Chapman, Chapman, \& Mishlove, 1982). It is possible that any decrease in positive affect for people with social anhedonia could be restricted to social situations. Moreover, given evidence that people with social anhedonia might have poorer quality social interactions (Collins, Blanchard, \& Biondo, 2005; possibly because of reduced emotional expression, Aghevli, Blanchard, \& Horan, 2003), any decrease in positive affect for social situations might be because people with social anhedonia have fewer interactions with people whom they are close to.

A third methodological factor is whether emotion reports are for retrospective or for current situations. Previous research has found some dissociations between how people remember feeling in previous situations versus how they actually report feeling in a current situation (Robinson \& Clore, 2002). For example, perhaps people with anhedonia have a memory bias and remember positive experiences as being less positive than they actually were (e.g., Horan et al., 2006, with a memory bias possibly developing because of experiencing fewer positive situations). In contrast, people with anhedonia may not differ in current or in-the-moment reports of emotion.

A fourth methodological factor is whether emotion reports are for emotion intensity or for emotion frequency (Schimmack \& Diener, 1997). People can vary in how intensely (or strongly) they experience emotions (e.g., tending to feel emotions like excited or serene weakly or strongly). At the same time, people can also vary in how frequently they experience emotions (e.g., tending to feel emotions like excited or serene rarely or quite often). If people with anhedonia experience more or less of a certain type of emotion-eliciting situation, it is possible that this could be reflected 
in a change only in emotion frequency but not in intensity (e.g., experiencing more negative situations and therefore having more frequent negative emotions).

A fifth methodological factor is whether emotion experience is assessed through direct self-report or is assessed indirectly. Previous research has found evidence that self-reported emotional experience may not be commensurate with other indicators of emotions. Hence, people might report not experiencing an emotion even when they behaviorally appear to be emotional (e.g., Berenbaum \& Irvin, 1996). Therefore, it would be helpful to assess emotional experience without directly asking participants to report their own emotions. For example, it has been found that people who on a questionnaire report reduced trait-level affect intensity also report less emotional content and focus less on emotions in their descriptions of life events (e.g., describing what it would be like to have your house burn down; Larsen, Billings, \& Cutler, 1996). However, if in anhedonia self-report is not an accurate reflection of emotional experience, then it is possible that a decrease in selfreported emotion might not be accompanied by decreased content in their descriptions of what it is like to experience life events.

Overall, in two studies we examined whether anhedonia was associated with changes in facets of emotional experience. For valence, we examined whether anhedonia was associated with decreased positive affect and increased negative affect. For arousal, we examined whether anhedonia would be associated with decreased high-arousal emotions. In addition, we used a variety of methods to characterize the nature of any change of emotional experience in anhedonia. We examined two possibilities for how emotional experience might be altered in anhedonia. One possibility is that anhedonia involves a generalized change in emotional experience, which predicts that differences in emotions should be found across situations and reporting formats. In contrast, the second possibility is that anhedonia involves a change in the types of experiences that people tend to have, which should be selectively reflected in changes in emotional experience for one or more of the following: retrospective (but not current) emotions, daily life (but not lab) situations, social (but not nonsocial) situations, frequency (but not intensity) of emotions, and direct self-reports (but not verbal descriptions) of emotional experience.

\section{Study 1}

In Study 1, we specifically examined social anhedonia. We did this because social anhedonia has been found to predict future schizophrenia-spectrum disorders (Gooding et al., 2005; Kwapil, 1998) and because to our knowledge few if any studies have examined self-reports of emotional experience in social anhedonia. In Study 1, we used an extreme-groups approach (Preacher, Rucker, MacCallum, \& Nicewander, 2005) that compared people with elevated social anhedonia with two other groups: a control group and a group with elevated levels of perceptual aberration and magical ideation (PerMag). We included the PerMag group to examine whether any changes in emotional experience would be specific to social anhedonia or would also be found in another group at increased risk of psychosis (L. J. Chapman, Chapman, Kwapil, Eckblad, \& Zinser, 1994).
Method

\section{Participants}

Participants were college students attending a large midwestern public university who received credit for an Introduction to Psychology course for their participation. They were selected from among a group of students $(n=4,165)$ who participated in departmental mass testing sessions in which they completed 15 items from the Revised Social Anhedonia Scale, seven items from the Perceptual Aberration Scale, and eight items from the Magical Ideation Scale. Individuals who scored either 2.0 standard deviations above or 0.5 standard deviations below the mass-testing same-sex gender mean were recruited for an individual testing session. At the individual testing session, participants completed the full versions of these scales, and participants for the current study were selected on the basis of their scores for the full version of the scale, with means and standard deviation cut-offs for the scales based on data obtained from a previous large-sample study (Kerns \& Berenbaum, 2000).

There were 40 people in the social anhedonia (SocAnh) group (27 women and 14 mean; mean age $=18.7, S D=1.2 ; 35$ Caucasian, 3 African American, and 2 Asian American) who scored at least 1.96 standard deviations above the same-sex mean on the Revised Social Anhedonia Scale. There were 30 people in the control group (20 women and 10 men; mean age $=18.6, S D=$ 1.1; 28 Caucasian, 1 African American, and 1 Asian American) who scored less than 0.5 standard deviation above the mean on the Revised Social Anhedonia, Perceptual Aberration, and Magical Ideation scales. There were 29 people in the PerMag group (17 women and 13 men; mean age $=18.6, S D=1.2 ; 26$ Caucasian, 2 African American, and 1 Asian American) who scored at least 1.96 standard deviation above the same-sex sample mean on either the Perceptual Aberration or the Magical Ideation scale or who had summed standardized Perceptual Aberration and Magical Ideation scores of 3 or higher (L. J. Chapman et al., 1994). Participants ( $n=$ 8) who scored high enough for both the SocAnh and the PerMag groups were excluded.

\section{Psychosis-Proneness and Personality Questionnaires}

Psychosis-proneness scales. Participants completed a 118item true-false questionnaire composed of a random mixture of all items of three psychosis-proneness scales-the Revised Social Anhedonia Scale (Eckblad et al., 1982), the Perceptual Aberration Scale (L. J. Chapman, Chapman, \& Raulin, 1978), and the Magical Ideation Scale (Eckblad \& Chapman, 1983)_as well as the Chapman Infrequency Scale (L. J. Chapman \& Chapman, 1983), which measures careless or invalid responses. Following previous research (e.g., Chmielewski, Fernandes, Yee, \& Miller, 1995), participants who endorsed three or more Chapman Infrequency Scale items were excluded. These scales have been used extensively in previous research (e.g., Edell, 1995; Horan, Blanchard, Gangstead, \& Kwapil, 2004; Lenzenweger, 1999). High scorers on the Social Anhedonia scale are at increased risk for schizophreniaspectrum disorders (Gooding et al., 2005; Kwapil, 1998), and high scorers on the Perceptual Aberration and Magical Ideation scales have been found to be at increased risk for future psychosis (L. J. Chapman et al., 1994). 
Personality traits. To examine whether any changes in emotion experience in anhedonia might be related to personality, the personality traits of extraversion and neuroticism were measured using the International Personality Item Pool (Goldberg, 1999), with 10 items for both Extraversion (e.g., "Am the life of the party") and Neuroticism (e.g., "Get stressed out easily"). Responses are made on a 5-point scale indicating amount of agreement. Although we examined whether personality could statistically account for emotional changes in anhedonia, it is unclear from previous research whether broad personality traits should be considered as the cause or the result of affective changes, making it uncertain whether they are a source of potential confounding (for methodological limitations of using statistical controls, see Miller \& Chapman, 2001).

\section{Emotional Experience}

Emotional experience was directly assessed in three different ways: the day reconstruction method (DRM), the situation rating task, and the picture rating task. In all three assessments, following Barrett and Russell (1999), 16 different emotions were rated, including positive high arousal (happy, excited, alert, and elated), positive low arousal (relaxed, contented, serene, and calm), negative high arousal (stressed, nervous, upset, and tense), and negative low arousal (lethargic, fatigued, sad, and depressed). Hence, this allowed for the assessment of both emotional valence (positive vs. negative) and emotional arousal (high vs. low; in addition, at the end of the study, we used these emotion terms to assess current mood).

In addition, in all three assessments, to separately assess emotion frequency and emotion intensity, we followed the procedures used by Schimmack and Diener (1997). Emotions were rated on a scale ranging from 0 to 6 . Participants were told that they should first decide whether they experienced a particular emotion or not and that if they did not experience that emotion that they should rate the emotion a 0 . Hence, the measure of affect frequency is the proportion of all possible emotions not rated 0 . Participants were told that if they had experienced the emotion, they were to rate how intensely they experienced that emotion on a scale ranging from 1 to 6 . Hence, the measure of intensity is the average of all emotions that were rated between 1 and 6 .

The DRM was developed by Kahneman, Krueger, Schkade, Schwarz, and Stone (2004) as a one-time assessment of emotional experience that would share the advantages of experience sampling methods (e.g., reduced memory bias, and therefore more likely to reflect actual emotional experience). On the DRM, people first reconstruct everything that happened to them on the previous day, from when they woke up until when they went to bed. People divide up their day into discrete episodes (e.g., getting ready for school, being in class, and going out to eat). After dividing up their day into episodes, they then rate how they felt during each episode. In addition, for each episode, people indicate whether they were with anyone and whom they were with (e.g., a friend or a significant other). This allows for an assessment of emotion in both social and nonsocial situations. It also allows for the assessment of emotion when interacting with people the participant is close to, defined as friends, significant others, and family. The DRM takes approximately 30-45 min to complete. On the DRM, the number of episodes into which participants divide their previous day can vary from person to person (e.g., in this study, the range was from 5 to 29). The three groups did not differ in the mean number of episodes on the DRM that they rated $(p=.60)$. In addition, as they were finishing the DRM, participants rated how typical their previous day was (from much worse, to typical, to much better). The groups did not differ significantly in how typical they rated the previous day $(p=.58)$.

In addition to the DRM, participants also completed the situation rating task. This method has also been recommended by Kahneman et al. (2004) as a one-time assessment of emotional experience that shares some of the advantages of experience sampling methods. Participants were first asked to remember the last time they were in a particular situation (excluding the previous day). Then, after they remembered a specific occasion, they then rated how they felt in that situation. Participants rated 20 different positive, neutral, and negative situations (last time they took an exam, went to a sporting event, went to a movie, talked to a friend on the phone, were in the car for more than $30 \mathrm{~min}$, read a book, watched a TV program you usually like, watched news on TV, went shopping, talked to a relative, talked to someone you found attractive romantically, exercised, ate at a restaurant, went to a party, attended class, went to the library, went to a coffee shop, had a disagreement with a friend, heard a joke, and played with an animal/pet). Compared with the DRM, the situation rating task can involve less recent experiences; hence, it is more susceptible to memory biases in people's ratings. However, one advantage of the situation rating task is that each participant rates his or her emotion over a broad range of experiences.

In addition to the DRM and the situation rating task, participants also completed a picture-rating lab task. On this task, participants saw a picture from the International Affective Picture Set (IAPS; Lang, Bradley, \& Cuthbert, 2005) for $10 \mathrm{~s}$. They then rated their emotional reaction to the picture. The picture-rating lab task complements the DRM and the situation rating task in two ways. On one hand, this task involves people's concurrent assessment of their emotional reactions. Hence, this assessment method eliminates any memory distortion in reporting emotional experience. On the other hand, every participant rates his or her emotional reactions to identical stimuli. Hence, any group differences cannot be because of differences in the events or stimuli that people experience. Participants rated 16 different IAPS pictures. The IAPS pictures used were the same ones selected by Barrett (2004) to assess a range of both emotional valence and emotional arousal. In the Results section, we present results for level of positive affect reported for positive pictures and for level of negative affect reported for negative pictures. However, there were no significant between-group differences for any of the other emotion ratings (all $p \mathrm{~s}>.45$; e.g., the groups did not differ in level of positive affect for negative or neutral pictures).

\section{Event Description Questionnaire}

Emotional experience was indirectly assessed with the Event Description Questionnaire (EDQ; Larsen et al., 1996; we used EDQ Version 1 in this study). On the EDQ, people are given eight events that they need to describe, four positive and four negative, with the events varying in how strongly they elicit affect (e.g., having your house burn down or losing your favorite pen). Participants are given $90 \mathrm{~s}$ to describe each event. They are told to 
imagine what each experience is like and then write down a description that would inform another person who has never had the experience before what it is like. Two ratings were made from the EDQ verbal descriptions. One, labeled emotional content (Larsen et al., 1996), is a count of the number of emotion-related terms within each description. The second, labeled focus on feelings (Larsen et al., 1996), is the degree to which people emphasize feelings versus facts in their description of the event, which is scored on a scale ranging from 1 (extreme focus on facts; e.g., for having money problems, "Having money problems does not enable you to do very much. You have to rely a lot on what you already have. You can't spend any money that you have on anything that isn't of important use") to 7 (extreme focus on feelings; e.g., for having money problems, "Anxiety, worry, this is a horrible feeling. It's a feeling of failure. You worry all of the time about people coming and taking away your possessions"). Ratings were made by two research assistants who were unaware of the hypotheses and of group membership. Using an intraclass correlation (Shrout \& Fliess, 1979) and treating the raters as random effects and the mean of the two raters as the unit of reliability, interrater reliability was .97 for positive emotional content, .87 for positive focus on feelings, .97 for negative emotional content, and .90 for negative focus on feelings. Total scores for emotional content and focus on feelings were highly associated with each other (for positive descriptions, $r=.88$; for negative description, $r=.94)$. Hence, a single positive event description variable and a single negative event description variable were created by summing standardized scores. In addition, the groups did not differ in the number of words in their descriptions, $F(2$, 96) $=0.39, p=.68$; for the SocAnh group, $M=33.5$ ( $S D=$ 13.7); for the PerMag group, $M=32.9(S D=16.4)$; and for the control group, $M=35.1$ ( $S D=14.9$; note also that the focus on feelings ratings are not based on the number of words produced).

\section{Procedure and Data Analysis}

Participants completed the study in the following order: EDQ, DRM, situation rating task, picture rating task, questionnaire measures, and current mood assessment. The EDQ was given first because pilot testing indicated that prior emotion ratings (e.g., on the DRM) could inadvertently increase the amount of emotional content provided on the EDQ (note also that because the DRM first involves reconstructing one's previous day, this means that there was an approximately 10- to 20-min delay between completing the EDQ and making emotion ratings on the DRM). In addition, note that if current mood assessment is largely influenced by prior emotion rating tasks, then (a) between-group results for current mood and emotion rating tasks should be the same and (b) current mood should statistically account for group differences in emotion rating tasks.

In analyzing the three emotion experience tasks (i.e., DRM, situation rating, and picture rating), we used 2 (valence: positive vs. negative) $\times 2$ (arousal: high vs. low) $\times 3$ (task: DRM vs. situation rating vs. picture rating) $\times 3$ (group: SocAnh vs. control vs. PerMag) mixed-model (three within-subjects factors and one between-subjects factor) analysis of variance (ANOVA). If the SocAnh group differed from the other two groups in positive affect but not in negative affect (or vice versa), this should be reflected in a significant Valence $\times$ Group interaction. Hence, significant
Valence $\times$ Group interactions were followed up by ANOVAs including only positive affect and including only negative affect (i.e., Arousal $\times$ Task $\times$ Group). At the same time, if the SocAnh group differed from the other groups in reports of emotion experience only for events in their daily lives but not for the picturerating lab task in which all participants saw the exact same stimuli, this should be reflected in a significant Task $\times$ Group interaction. Significant omnibus results were followed up with planned comparisons between (a) the SocAnh group and the Control group and (b) the SocAnh group and the PerMag group. To control for the familywise error rate, for these planned comparisons we used a Bonferroni correction, with a significance level of $p<.025$.

In reporting effect sizes, we used partial eta-square for omnibus tests and point-biserial correlations for all other effect sizes (for point-biserial correlation, see Rosenthal, 1991; note that $r_{\mathrm{pb}}$ can be converted to $d$, with $r_{\mathrm{pb}} \geq 0.371, r_{\mathrm{pb}} \geq 0.243$, and $r_{\mathrm{pb}} \geq 0.10$ corresponding to large, medium, and small effect sizes, respectively). Positive $r_{\mathrm{pb}}$ effect sizes indicate larger effects for the SocAnh group than for the other groups (or larger for the PerMag group than for the control group).

\section{Results}

\section{Intensity of Emotion}

For intensity of emotion ratings, as can be seen in Table 1, there was a significant interaction between valence and group, $F(2$, 96) $=6.04, p=.003$, partial $\eta^{2}=0.118$. The interaction between group and valence reflects that there was a significant betweengroups difference in an ANOVA for positive affect but not for negative affect: positive affect, $F(2,96)=4.45, p=.014$, partial $\eta^{2}=0.083$; negative affect, $F(2,96)=0.27, p=.973$, partial $\eta^{2}=0.001$. We next compared the SocAnh group with the other two groups on positive affect intensity. The SocAnh group reported significantly less intense positive affect did than the control group, $t(68)=3.51, p=.0008, r_{\mathrm{pb}}=-.39$, and the PerMag group, $t(67)=2.38, p=.02, r_{\mathrm{pb}}=-.28$ (note that the effect size

Table 1

Intensity of Positive Affect (PA) and Negative Affect (NA) by Arousal Level and by Task in Study 1

\begin{tabular}{lccc}
\hline \multicolumn{1}{c}{ Measure } & SocAnh & PerMag & Control \\
\hline Day reconstruction method & & & \\
PA, high arousal & $2.80(0.87)$ & $3.23(0.96)$ & $3.16(0.61)$ \\
PA, low arousal & $2.72(0.82)$ & $2.93(1.09)$ & $3.23(0.80)$ \\
NA, high arousal & $2.37(0.99)$ & $2.43(0.83)$ & $2.41(0.87)$ \\
NA, low arousal & $2.73(0.77)$ & $2.29(0.94)$ & $2.52(0.73)$ \\
Situation rating task & & & \\
PA, high arousal & $3.54(0.71)$ & $3.90(0.87)$ & $4.04(0.67)$ \\
PA, low arousal & $3.25(0.70)$ & $3.48(0.91)$ & $3.71(0.85)$ \\
NA, high arousal & $2.85(0.77)$ & $2.95(0.77)$ & $2.93(0.76)$ \\
NA, low arousal & $2.64(0.76)$ & $2.65(0.86)$ & $2.65(0.79)$ \\
Picture rating task & & & \\
PA, high arousal & $3.73(0.98)$ & $3.95(0.94)$ & $4.23(0.95)$ \\
PA, low arousal & $3.54(0.93)$ & $3.95(0.84)$ & $4.06(1.01)$ \\
NA, high arousal & $3.89(1.10)$ & $4.06(1.22)$ & $3.90(1.12)$ \\
NA, low arousal & $3.47(1.01)$ & $3.59(1.12)$ & $3.63(1.14)$ \\
\hline
\end{tabular}

Note. Standard deviations appear in parentheses. SocAnh $=$ social anhedonia group; PerMag $=$ perceptual aberration and magical ideation group. 
difference between the PerMag group and the control group was $-.10)$. For positive affect intensity, the interaction between task and group was not significant, $F(2,96)=0.18, p=.84$, partial $\eta^{2}=0.004$ (i.e., the SocAnh group reported decreased positive affect intensity on all three tasks; e.g., comparing SocAnh and Controls on positive affect intensity, between-groups effect sizes for the three tasks were $-.30,-.33$, and -.27$)$. No other effects involving group were significant (all $p \mathrm{~s}>$.35). For example, the interaction between group and arousal was not significant, $F(2$, 96) $=1.04, p=.36$, partial $\eta^{2}=0.024$, as the SocAnh group did not selectively report fewer high-arousal emotions than did controls (i.e., results were similar for high- and low-arousal emotions; e.g., the between-groups effect sizes between the SocAnh and the control groups for positive affect were -.31 for high arousal and -.34 for low arousal; for negative affect they were -.02 for high arousal and .01 for low arousal).

Given that the SocAnh group reported decreased positive affect intensity for events in their daily lives, one possible explanation for this decrease is that the SocAnh group spends less time with people they are close to. To attempt to examine this, we examined positive affect intensity ratings on the DRM only for social situations in which people were with friends, significant others, or family. Overall, there was a significant between-groups difference in positive affect intensity, $F(2,96)=3.33, p=.04$, partial $\eta^{2}=$ 0.083 , as even for these close relationships the SocAnh group reported significantly decreased positive affect intensity than did the control group, $t(68)=2.90, p=.005, r_{\mathrm{pb}}=-.33$, but they did not differ significantly from the PerMag group, $t(67)=1.36, p=$ $.18, r_{\mathrm{pb}}=-.16$. In addition, the groups did not significantly differ in the number of situations in which they interacted with people they were close to, $F(2,96)=0.83, p=.44$; for the SocAnh group, $M=6.2(S D=3.1)$; for the PerMag group, $M=6.5(S D=$ $3.0)$; and for the control group, $M=7.2(S D=3.6)$.

Another possible explanation for the decrease in positive affect intensity for daily experiences in the SocAnh group is that it is limited to social situations. If true, this would predict that those in the SocAnh group should not report decreased positive affect intensity when they are alone. As can be seen in Figure 1, there was a trend for a significant Arousal $\times$ Group interaction, $F(2$, 96) $=2.84, p=.06$, partial $\eta^{2}=0.066$. This trend for an interaction reflects that for situations in which they were alone, the SocAnh and control groups differed on low-arousal positive affect intensity, $t(68)=2.41, p=.019, r_{\mathrm{pb}}=-.28$, but not on high-

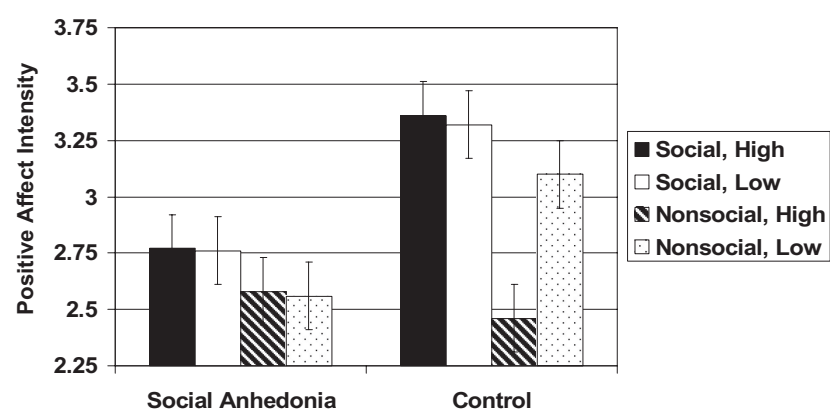

Figure 1. Positive affect intensity for high-arousal emotions and lowarousal emotions and for social and nonsocial situations in the social anhedonia and control groups on the day reconstruction method in Study 1. arousal positive affect intensity, $t(68)=0.24, p=.81, r_{\mathrm{pb}}=.03$ (the control group, as can be seen in Figure 1, reported decreased high-arousal positive emotions, such as excited, when they were alone compared with when they were with others). In addition, the groups did not significantly differ in the number of situations in which they were alone: $F(2,96)=0.99, p=.37$; for the SocAnh group, $M=4.8(S D=3.2)$; for the PerMag group, $M=5.4(S D=$ $3.1)$; and for the control group, $M=4.3(S D=2.0)$.

\section{Frequency of Emotion}

For frequency of emotion ratings, as can be seen in Table 2, there was a significant interaction between valence and group, $F(2,96)=$ 5.30, $p=.0066$, partial $\eta^{2}=0.103$. The interaction between group and valence reflects that there was a significant between-groups difference in an ANOVA for negative affect but not for positive affect: negative affect, $F(2,96)=3.41, p=.037$, partial $\eta^{2}=0.067$; positive affect, $F(2,96)=1.21, p=.30$, partial $\eta^{2}=0.026$. We next compared the SocAnh group with the other two groups on negative affect frequency. There was a trend for the SocAnh group to report significantly more frequent negative affect than the control group, $t(68)=2.24, p=.028, r_{\mathrm{pb}}=.26$ (again, Bonferroni corrected significant $p<.025)$. In contrast, the SocAnh and PerMag groups did not differ significantly on negative affect frequency, $t(67)=0.44, p=$ $.66, r_{\mathrm{pb}}=-.05$ (note that the effect size difference between the PerMag and control groups was .30). In addition, for negative affect frequency, there was trend for a significant interaction between task and group, $F(2,96)=0.18, p=.068$, partial $\eta^{2}=0.046$. As can be seen in Table 2, this interaction reflects that the SocAnh group differed from controls in negative affect frequency in reporting on emotions for events in their daily lives, $t(68)=2.67, p=.01, r_{\mathrm{pb}}=$ .31 (note the effect size for the difference between the PerMag and control groups was .35); however, the SocAnh group did not differ from controls in negative affect frequency on the picture-rating lab task, $t(68)=0.46, p=.65, r_{\mathrm{pb}}=.06$ (note that the effect size for the difference between the PerMag and control groups was .12). No other effects involving group were significant (all $p \mathrm{~s}>.30$ ). For example, the interaction between group and arousal was not significant, $F(2$, 96) $=1.03, p=.36$, partial $\eta^{2}=0.023$, as the SocAnh group did not appear to report selectively fewer high-arousal emotions than controls (i.e., results were similar for high- and low-arousal emotions; e.g., the between-groups effect sizes between the SocAnh and control groups for positive affect were -.12 for high arousal and -.13 for low arousal; for negative affect, they were .22 for high arousal and .24 for low arousal).

\section{Verbal Descriptions of Emotional Experience}

To analyze verbal descriptions of emotion-eliciting events on the EDQ, we analyzed data using a 2 (valence: positive vs. negative) $\times$ 3 (group: SocAnh vs. control vs. PerMag) mixed-model ANOVA. Overall, there was a significant between-groups difference in the amount of emotional content, $F(2,96)=3.70, p=.028$, partial $\eta^{2}=$ .072. As can be seen in Table 3, this overall between-groups difference reflected that the SocAnh group provided significantly less emotional content and focused significantly less on feelings than the PerMag group, $t(67)=2.76, p=.007, r_{\mathrm{pb}}=-.32$, and a trend for the SocAnh group to report less emotional content than the control group, $t(68)=2.04, p=.045, r_{\mathrm{pb}}=-.24$. Given previous and 
Table 2

Frequency of Positive Affect (PA) and Negative Affect (NA) by Arousal Level and by Task in Study 1

\begin{tabular}{cccc}
\hline Measure & SocAnh & PerMag & Control \\
\hline Day reconstruction method & & & \\
PA, high arousal & $0.59(0.23)$ & $0.68(0.18)$ & $0.62(0.25)$ \\
PA, low arousal & $0.67(0.26)$ & $0.73(0.20)$ & $0.72(0.23)$ \\
NA, high arousal & $0.47(0.27)$ & $0.51(0.28)$ & $0.34(0.21)$ \\
NA, low arousal & $0.45(0.25)$ & $0.44(0.27)$ & $0.31(0.24)$ \\
Situation rating task & & & \\
PA, high arousal & $0.74(0.17)$ & $0.80(0.12)$ & $0.76(0.17)$ \\
PA, low arousal & $0.76(0.16)$ & $0.79(0.15)$ & $0.77(0.19)$ \\
NA, high arousal & $0.47(0.25)$ & $0.50(0.20)$ & $0.33(0.20)$ \\
NA, low arousal & $0.40(0.25)$ & $0.42(0.21)$ & $0.29(0.23)$ \\
Picture rating task & & & \\
PA, high arousal & $0.72(0.21)$ & $0.79(0.18)$ & $0.73(0.26)$ \\
PA, low arousal & $0.78(0.19)$ & $0.83(0.15)$ & $0.79(0.23)$ \\
NA, high arousal & $0.72(0.22)$ & $0.77(0.25)$ & $0.73(0.24)$ \\
NA, low arousal & $0.52(0.21)$ & $0.53(0.22)$ & $0.49(0.23)$ \\
\hline
\end{tabular}

Note. Standard deviations appear in parentheses. SocAnh $=$ social anhedonia group; PerMag = perceptual aberration and magical ideation group.

current evidence that social anhedonia reflects decreased positive affect, we explored whether the SocAnh group would differ from controls for both positive events and negative events. The SocAnh group did provide less emotional content and focused less on feelings than the control group for descriptions of positive events, $t(68)=$ $2.41, p=.019, r_{\mathrm{pb}}=-.28$, but not for descriptions of negative events, $t(68)=1.02, p=.31, r_{\mathrm{pb}}=-.12$.

\section{Relationship Between Personality, Current Mood, and Emotion Experience Ratings}

As can be seen in Table 3 for personality, compared with the control group the SocAnh group reported significantly lower levels of extraversion, $t(68)=7.51, p=.000001, r_{\mathrm{pb}}=-.67$, and higher levels of neuroticism, $t(68)=2.89, p=.005, r_{\mathrm{pb}}=.33$. In addition, as can be seen in Table 3 for current mood, the SocAnh group tended to report lower positive affect, $t(68)=2.04, p=$

Table 3

Event Description Questionnaire, Personality, and Current Mood in Study 1

\begin{tabular}{lrrr}
\hline \multicolumn{1}{c}{ Measure } & \multicolumn{1}{c}{ SocAnh } & PerMag & \multicolumn{1}{c}{ Control } \\
\hline Event description questionnaire & & & \\
$\quad$ Positive emotional content & $1.9(0.84)$ & $2.5(1.30)$ & $2.4(1.16)$ \\
Positive focus on feeling & $3.3(1.06)$ & $4.0(1.28)$ & $3.9(1.16)$ \\
Positive event description & $-0.34(0.83)$ & $0.26(1.15)$ & $0.20(1.01)$ \\
Negative emotional content & $1.9(0.92)$ & $2.5(0.96)$ & $2.1(1.07)$ \\
Negative focus on feeling & $3.4(1.24)$ & $4.2(1.25)$ & $3.7(1.30)$ \\
$\quad$ Negative event description & $-0.26(0.97)$ & $0.37(0.98)$ & $-0.02(1.06)$ \\
Personality & & & \\
$\quad$ Extraversion & $2.56(0.86)$ & $3.62(0.91)$ & $3.82(0.46)$ \\
$\quad$ Neuroticism & $3.12(0.88)$ & $3.04(0.76)$ & $2.52(0.83)$ \\
Current Mood & & & \\
$\quad$ Positive affect & $1.7(1.04)$ & $1.9(1.15)$ & $2.2(1.01)$ \\
Negative affect & $1.9(1.22)$ & $2.1(1.37)$ & $1.3(0.73)$ \\
\hline
\end{tabular}

Note. Standard deviations appear in parentheses. SocAnh $=$ social anhedonia group; PerMag = perceptual aberration and magical ideation group.
$.045, r_{\mathrm{pb}}=-.24$, but significantly higher negative affect, $t(68)=$ $2.41, p=.019, r_{\mathrm{pb}}=.28$, than the control group. We next examined whether personality and current mood could statistically account for differences between the SocAnh and the control groups in positive affect intensity. For this analysis, we computed a single composite positive affect intensity score by averaging scores on the three emotion experience tasks. As can be seen in Table 4 , in a hierarchical multiple regression, personality and current mood were entered first on Step 1. On Step 2, after statistically accounting for variance shared with personality and current mood, categorical group membership (i.e., SocAnh vs. control) still significantly predicted decreased positive affect intensity (in addition, statistically accounting for variance shared with positive affect frequency did not affect group differences in positive affect intensity).

\section{Study 2}

There were five main goals for Study 2. Study 1 found that people with elevated social anhedonia reported decreased positive affect intensity. However, in addition to social anhedonia, people with schizophrenia also report elevated physical anhedonia (L. J. Chapman et al., 1976). One main goal of Study 2 was to examine, in addition to social anhedonia, whether physical anhedonia would also be associated with decreased positive affect intensity.

A second goal of Study 2 was to examine whether current distress could statistically account for associations between anhedonia and positive affect intensity. Previous research has found evidence that self-reported pleasure is reduced in people currently under stress (e.g., final exam week; Berenbaum \& Connelly, 1993). From this view, perhaps people with elevated anhedonia in Study 1 reported decreased positive affect intensity because they were currently under stress. Hence, Study 2 examined whether associations between anhedonia and positive affect intensity could be accounted for by current levels of self-reported depression or by reports of recent major or minor stressors.

A third goal of Study 2 was to further examine whether decreased positive affect intensity in anhedonia was not statistically accounted for by perceptual aberration-magical ideation, personality, or current mood. In Study 1, these other variables did not

Table 4

Summary of Hierarchical Regression Analyses for Variables Predicting Positive Affect Intensity in Study $1(N=70)$

\begin{tabular}{lrrr}
\hline \multicolumn{1}{c}{ Variable } & $B$ & $S E B$ & $\beta$ \\
\hline Step 1 & & & \\
$\quad$ Extraversion & .14 & .15 & 0.16 \\
Neuroticism & -.11 & .13 & -0.14 \\
Current positive affect & .27 & .12 & $0.28^{*}$ \\
Current negative affect & .12 & .12 & 0.15 \\
Step 2 & & & \\
Extraversion & .09 & .15 & 0.07 \\
Neuroticism & -.05 & .12 & -0.04 \\
Current positive affect & .21 & .12 & $0.25^{*}$ \\
Current negative affect & .17 & .11 & 0.20 \\
Group (social anhedonia vs. control) & -.54 & .21 & $-0.36^{* * *}$ \\
\hline
\end{tabular}

Note. $R^{2}=.21$ for Step $1, p<.05 ; \Delta R^{2}=.09$ for Step $2, p<.01$. ${ }^{*} p<.05$. ${ }^{* * *} p<.01$. 
statistically account for decreased positive affect intensity in social anhedonia. However, some variables were measured categorically, and other variables were measured dimensionally. The influence of one variable on another could potentially be more clearly examined when they are all measured in a similar way (Kerns, 2006). In Study 2, we separately and dimensionally measured both anhedonia and perceptual aberration-magical ideation to further assess whether only anhedonia and not psychosis proneness in general was associated with decreased positive affect intensity. At the same time, we further examined whether decreased positive affect intensity in anhedonia was not statistically accounted for either by personality or by current mood.

A fourth goal of Study 2 was to examine whether in a separate set of participants anhedonia would again be associated with decreased self-reported positive affect intensity for lab stimuli. Study 1 found that people with elevated social anhedonia report decreased intensity of positive affect both for reports of real-world experiences and for responses to lab stimuli. In contrast, research on people with schizophrenia has generally not found decreased self-reported positive affect in response to lab stimuli (e.g., Burbridge \& Barch, 2007). Therefore, Study 2 examined whether the finding of an association between anhedonia and decreased positive affect intensity for lab stimuli would replicate in a new sample.

A fifth goal of Study 2 was to examine whether anhedonia would be associated with decreased positive affect for lab stimuli using a dimensional design. An important advantage of dimensional designs is that they provide a less biased estimate of the effect size for the association between two variables than an extreme-group design that selects groups from the extreme tails of the distribution (Preacher et al., 2005). In fact, it is possible that variation in control group selection could partially account for some of the variation in previous research on positive affect in physical anhedonia. Overall, two previous published studies did not find that physical anhedonia is associated with decreased positive affect for positive lab stimuli (Berenbaum et al., 1987; Germans \& Kring, 2000). In contrast, three previous published studies did find a decrease in physical anhedonia (Ferguson \& Katkin, 1996; Fiorito \& Simons, 1994; Fitzgibbons \& Simons, 1992). More important, the studies that did find a decrease in physical anhedonia probably selected a more extreme control group than the two studies that did not find a decrease. Hence, variation in whether anhedonia is associated with decreased positive affect for lab stimuli might be because of variation in the selection of the control group. In Study 2, we measured anhedonia dimensionally and therefore provide an estimate of the association between anhedonia and positive affect that is less biased relative to an extreme-group design.

In Study 1, social anhedonia was measured categorically in an extreme-groups design. In Study 2, social and physical anhedonia were measured dimensionally. Each study design has potential pros and cons. On one hand, categorical designs might be a more accurate way of assessing schizotypy facets if they are truly categorical variables (Horan et al., 2004), some schizotypy measures were initially designed to measure the extreme end of the distribution that is focused on in an extreme-groups design, and there is potentially much less information in the literature about the relationship between measures of schizotypy with other variables across the entire range of schizotypy scores. On the other hand, dimensional designs might allow for a more straightforward examination of the specificity of the association with a schizotypy facet when removing variance shared with other variables. At the same time, previous research has found some similar results for schizotypy facets whether measured dimensionally or categorically (e.g., Kerns, 2005, 2006; Kerns \& Becker, 2008; Martin \& Kerns, 2008a, 2008c), and with large enough samples dimensional designs can still allow for a categorical analysis of data. Perhaps most important, as previously discussed, dimensional studies provide a less biased measure of effect size (Preacher et al., 2005). Overall, using both categorical and dimensional designs across multiple studies with their complementary strengths might be able to provide important converging evidence about schizotypy (Shadish, Cook, \& Campbell, 2002).

\section{Method}

\section{Participants}

Participants were 339 college students (187 women and 152 men; mean age $=18.7, S D=1.5 ; 89 \%$ Caucasian, $7 \%$ African American, 2\% Latino/Latina, and 2\% Asian American) attending a large midwestern public university who received credit for an Introductory Psychology course for their participation.

\section{Questionnaire Measures}

Psychosis-proneness scales. As in Study 1, participants completed the Revised Social Anhedonia Scale, the Perceptual Aberration Scale, the Magical Ideation Scale, and the Chapman Infrequency Scale. In addition, in Study 2 they also completed the Physical Anhedonia scale (L. J. Chapman et al., 1976). Using standardized scores, we created a single anhedonia score (summing level of social and physical anhedonia) and a single PerMag score (summing level of perceptual aberration and magical ideation). However, given that anhedonia scales tend to be only moderately correlated and have exhibited different associations with other variables in some previous research (e.g., Prince \& Berenbaum, 1993), we also report results separately for social and physical anhedonia.

Personality traits. As in Study 1, the personality traits of extraversion and neuroticism were measured using the International Personality Item Pool (Goldberg, 1999).

Current distress. Participants completed two measures to assess their current level of distress. One measure was the Beck Depression Inventory-Second Edition (A. T. Beck, Steer, \& Brown, 1996), a frequently used measure of current depression and psychological distress. The second measure was the Undergraduate Stress Questionnaire (Crandall, Preisler, \& Aussprung, 1992), which lists a number of stressors that could be experienced by undergraduate students ranging from major to minor stressors (e.g., "death of a family member or friend," "victim of a crime," "property stolen," "had a class presentation," and "got a traffic ticket"). Two measures of stress were calculated: (a) recent major stress, the frequency of major stressors reported in the previous 2 weeks, and (b) recent minor stress, the frequency of minor stressors reported in the previous 2 weeks.

\section{Emotional Experience}

Participants completed the same picture-rating task as in Study 1. 


\section{Procedure and Data Analysis}

Participants completed the study in the following order: picture rating, questionnaire measures, and current mood assessment (note that the picture-rating task was the last emotion experience task given in Study 1, but the first in Study 2). In data analysis, our primary focus was to examine two associations, whether (a) anhedonia or (b) PerMag scores would be associated with decreased positive affect intensity. In addition, in a hierarchical multiple regression, we examined whether anhedonia would significantly add to the prediction of positive affect intensity after statistically removing variance shared with other variables associated with anhedonia. Note that because use of extreme-groups designs (as in Study 1) inflates effect sizes (Preacher et al., 2005), estimates of the association between anhedonia and positive affect intensity in Study 2 were expected to be smaller than in Study 1 .

\section{Results}

As can be seen in Table 5, as found in Study 1 anhedonia was significantly associated with decreased positive affect intensity on the picture-rating task (anhedonia was associated with both higharousal positive emotions $[r=-.18, p=.001]$ and low-arousal positive emotions $[r=-.16, p=.004])$. At the same time, both social anhedonia $(r=-.14, p=.01)$ and physical anhedonia $(r=$ $-.20, p=.0002)$ were significantly associated with decreased positive affect intensity (moreover, if analyzed categorically, 28 people had elevated anhedonia, and they also differed significantly from control participants in level of positive affect intensity, $p<$ .01). In contrast to anhedonia, as can be seen in Table 5, PerMag scores were not associated with positive affect intensity. Instead, PerMag scores were significantly associated with increased negative affect intensity.

As can also be seen in Table 5, anhedonia was significantly associated with personality, current mood, and current levels of distress. Hence, we next examined whether these other variables, plus PerMag scores, could account for the association between anhedonia and positive affect intensity. In a hierarchical multiple regression, we first entered PerMag scores, personality, current

Table 5

Correlations Between Psychosis-Proneness Scores and Individual Differences in Study 2

\begin{tabular}{lll}
\hline \multicolumn{1}{c}{ Measure } & Anhedonia & $\begin{array}{l}\text { Perceptual aberration } \\
\text { and magical ideation }\end{array}$ \\
\hline Picture rating intensity & & \\
$\quad$ Positive & $-.20^{* *}$ & .03 \\
Negative & -.08 & $.11^{*}$ \\
Personality & $-.34^{* *}$ & .03 \\
$\quad$ Extraversion & $.18^{* *}$ & $.24^{* *}$ \\
Neuroticism & $-.16^{* *}$ & .01 \\
Current mood & $.12^{*}$ & $.25^{* *}$ \\
$\quad$ Positive & $.31^{* *}$ & $.43^{* *}$ \\
Negative & .06 & $.12^{*}$ \\
Current distress & .09 & $.41^{* *}$ \\
Beck Depression Inventory & & \\
Recent major stress & & \\
Recent minor stress & & \\
\hline * $p$.05. $p<.01$. & &
\end{tabular}

mood, and current distress. After this, we then entered anhedonia. As can be seen in Table 6, in this analysis anhedonia still significantly predicted decreased positive affect intensity.

\section{Discussion}

The goal of this research was to examine self-reported emotional experience in anhedonia, in particular the facets of valence and arousal. In addition, this research examined whether anhedonia was associated with a generalized change in emotional experience or whether self-reported emotional experience varied by types of experiences or reporting format. For valence, we found in two studies that anhedonia was associated with decreased positive affect intensity. Moreover, decreased intensity appeared to be generalized across both situations and reporting formats. For example, in Study 1 there was a decrease of positive affect intensity in people's daily lives, consistent with a recent experience sampling study (Brown, Silvia, Myin-Germeys, \& Kwapil, 2007). Decreased intensity was found even in situations in which people with social anhedonia were interacting with people they are close to. In addition, even for situations in which they were alone, people with elevated social anhedonia reported decreased positive affect intensity. At the same time, in both studies there was a decrease in positive affect intensity for lab stimuli. Hence, there was evidence of decreased positive affect intensity even when controlling for any differences in the types of situations experienced. Furthermore, the decreased intensity was for both retrospective and current reports of emotion, suggesting that the decrease for daily life situations probably cannot be entirely accounted for by poor memory for positive emotions, which is consistent with what has been found in schizophrenia (Horan et al., 2006). The decrease in Study 1 was for intensity but not for frequency of positive affect, providing some evidence that people with social anhedonia did not differ from controls in the number of positive events but in their reactions to events. In addition to self-reported emotion, in Study 1 anhedonia was associated with decreased emotional content and focus on feelings in verbal descriptions of what it is like to experience positive emotional events. Therefore, even when people with anhedonia were not directly asked to report their emotions, there was evidence of decreased positive affective content in their verbal behavior. Overall, the results of these two studies suggest that anhedonia in people at possible risk for schizophreniaspectrum disorders might be associated with a general decrease in the experience of positive affect intensity.

At the same time, there was evidence that decreased positive affect intensity might be specifically related to anhedonia. In both studies, there was evidence that decreased positive affect intensity was associated with anhedonia but not with a general increased risk of psychosis proneness, as perceptual aberration-magical ideation was not associated with positive affect in either study. Moreover, in both studies personality and current mood did not statistically account for the association between anhedonia and decreased positive affect intensity.

In contrast to positive affect, for negative affect there was evidence that anhedonia might be associated with an increased frequency of experiencing negative emotional situations and not with a generalized increase in the experience of negative emotions. In both studies, anhedonia was not associated with negative affect intensity. Instead, anhedonia was associated with increased fre- 
Table 6

Summary of Hierarchical Regression Analyses for Variables Predicting Positive Affect Intensity in Study $2(N=339)$

\begin{tabular}{lrrr}
\hline \multicolumn{1}{c}{ Variable } & $B$ & $S E B$ & $\beta$ \\
\hline Step 1 & & & \\
$\quad$ Extraversion & .11 & .05 & $0.12^{*}$ \\
Neuroticism & .12 & .06 & 0.12 \\
Current positive affect & .25 & .05 & $0.26^{* * *}$ \\
Current negative affect & .18 & .06 & $0.19^{\text {*** }}$ \\
Beck Depression Inventory & -.11 & .07 & -0.11 \\
Perceptual aberration and magical ideation & -.02 & .06 & -0.02 \\
Step 2 & & & \\
Extraversion & .07 & .05 & 0.08 \\
Neuroticism & .11 & .06 & 0.12 \\
Current positive affect & .23 & .05 & $0.25^{\text {*** }}$ \\
Current negative affect & .17 & .06 & $0.18^{\text {*** }}$ \\
Beck Depression Inventory & -.08 & .07 & -0.08 \\
Perceptual aberration and magical ideation & -.01 & .06 & -0.01 \\
Anhedonia & -.17 & .06 & $-0.16^{\text {*** }}$ \\
\hline
\end{tabular}

Note. $R^{2}=.12$ for Step $1, p<.001 ; \Delta R^{2}=.03$ for Step $2, p<.01$. ${ }^{*} p<.05$. *** $p<.01$.

quency of negative emotions. However, the increase in frequency was found only for daily life situations but not for lab stimuli, suggesting that the increase in negative affect frequency might be related to people with anhedonia experiencing more frequent negative daily life events. At the same time, the increase in negative affect frequency did not appear to be specific to anhedonia, as this was also found in perceptual aberration-magical ideation. This suggests that psychosis proneness or risk for psychopathology in general might be associated with increased reports of negative affect in daily life situations.

In contrast to valence, the current research did not find evidence that anhedonia is associated with a specific decrease in the experience of high-arousal emotions. Instead, to the extent that anhedonia was associated with changes in high-arousal emotions (e.g., a decrease in high-arousal positive affect intensity), anhedonia was similarly associated with changes in low-arousal emotions. Hence, this study does not support the hypothesis that anhedonia reflects a decrease specifically in the emotional experience of arousal. More important, self-reported emotional arousal is not synonymous with either physiological measures of emotion or with selfreports of physiological processes (e.g., detecting heart rate changes; Barrett et al., 2007). Therefore, it is possible that anhedonia might be associated with some other aspect of emotional arousal. However, the current research did not find evidence that anhedonia was associated with a decrease specifically in the self-report of higharousal emotions.

Hence, on the basis of the current research examining the facets valence and arousal, it appears that anhedonia is associated with decreased positive affect intensity. As mentioned previously, emotional experience is thought to involve certain core affective features, such as valence and arousal, and how these affective features are experienced depends on attention and conceptual processing (Barrett et al., 2007). Therefore, there are at least two possible explanations for the decrease in positive affect intensity in anhedonia. One explanation is that anhedonia reflects a decreased capacity to experience positive affect. The other explanation is that decreased positive affect intensity in anhedonia reflects decreased attention to and conceptual processing of positive affect.

On one hand, a decrease in the capacity to experience positive affect in anhedonia does seem generally consistent with the current finding of decreased positive affect intensity in anhedonia across a range of situations and reporting formats. On the other hand, a decrease in capacity might seem inconsistent with other research on positive affect for lab stimuli in people with schizophrenia. However, overall, previous research has found some evidence of a decrease in positive affect in people with schizophrenia, although the decrease may be quite small. For example, in 25 previous studies examining positive affect for lab stimuli, 20 of these studies reported numerically lower positive mood in people with schizophrenia (Cohen \& Minor, in press), a difference that is significant in a vote-counting meta-analysis using a sign test ( $p=.004$; Rosenthal, 1991). However, the effect size difference across these studies is quite small $\left(r_{\mathrm{pb}}>-.16\right.$, at least). However, it is possible that the effect size in people with schizophrenia might be at least somewhat larger if these studies focused specifically on people with anhedonia. For example, in the current research PerMag scores were not associated with decreased positive affect, consistent with the possibility that people with schizophrenia who do not have anhedonia may not report decreased positive affect after positive lab stimuli.

Although it is possible that people with anhedonia have a decreased capacity to experience positive affect, we think a more likely interpretation for the decrease in positive affect intensity in the current research is that it reflects a decreased attention to and conceptual processing of positive affect. Hence, from this view, although people with anhedonia could experience pleasure to the same extent as controls, they are less likely to pay attention to or to conceptually elaborate their feelings and are therefore less likely to experience them as specific positive emotions (Barrett et al., 2007; Frijda \& Sundararajan, 2007; Lambie \& Marcel, 2002). A decrease in attention to emotions in anhedonia is consistent with some previous anhedonia research. For example, anhedonia in people at possible risk for schizophrenia-spectrum disorders has been strongly associated with decreased scores on the attention to emotions questionnaire (Berenbaum et al., 2006; Kerns, 2006). At the same time, we have recently found in people with schizophrenia $(n=47)$ that clinically rated anhedonia is also strongly negatively associated $(r=-.50)$ with the attention to emotions questionnaire (Becker, Cicero, \& Kerns, 2007). More important, other research on the attention to emotions scale has found that it is specifically associated with attention to positive emotions (Gasper \& Bramesfeld, 2006). At the same time, in Study 1 decreased positive affect content and decreased focus on feelings on the EDQ also seems consistent with a decreased attention to and conceptual processing of positive emotions. Therefore, it is possible that the current finding of decreased positive affect intensity in anhedonia is related to decreased attention to and conceptual processing of positive affect.

A decrease in attention to and conceptual processing of positive affect also seems consistent with other research on motivation in schizophrenia. There is some evidence that people with schizophrenia report a decreased wanting but not a decreased liking of stimuli (Gard, Kring, Gard, Horan, \& Green, 2007). It is possible that decreased wanting and anticipation of positive stimuli could potentially contribute to decreased attention to and thinking about 
positive emotions. At the same time, it is possible that anhedonia might be associated with a generalized problem in the controlled processing of emotional, especially positive, stimuli. For example, we have recently found in two separate studies that social anhedonia is associated with poorer controlled processing of emotional stimuli (Martin \& Kerns, 2008b). Another possibility is that the length of stimulus processing could influence the depth of emotional processing (Frijda \& Sundararajan, 2007), which could also influence whether controls are more likely to report an increase in positive affect compared with people with anhedonia. In fact, one study (Germans \& Kring, 2000) that did not report a significant decrease in positive affect in people with elevated physical anhedonia used a shorter stimulus duration $(6 \mathrm{~s})$ than the studies that have found a significant decrease in positive affect $(\geq 10 \mathrm{~s}$; Ferguson \& Katkin, 1996; Fiorito \& Simons, 1994; Fitzgibbons \& Simons, 1992; current Study 1 and 2). At the same time, another issue for future research is to examine the relationship between attention to and conceptualization of positive emotions and reports of emotional experience in people with anhedonia.

Research on people at risk for schizophrenia-spectrum disorders can provide evidence about the nature of the liability for schizophrenia without many of the confounds involved in schizophrenia research (e.g., medication; Blanchard \& Neale, 1992). An issue for future research is to further examine whether people with schizophrenia report reduced intensity for specific positive emotions and whether it is related to decreased attention to emotions. At the same time, another issue for future research is to continue to compare similarities and differences between social and physical anhedonia. For example, future research could examine whether physical anhedonia is also associated with decreased positive affect intensity for people's daily experiences.

\section{References}

Aghevli, M. A., Blanchard, J. J., \& Horan, W. P. (2003). The expression and experience of emotion in schizophrenia: A study of social interactions. Psychiatry Research, 119, 261-270.

Barrett, L. F. (2004). Feelings or words? Understanding the content in self-report ratings of experienced emotion. Journal of Personality and Social Psychology, 87, 266-281.

Barrett, L. F., Mesquita, B., Ochsner, K. N., \& Gross, J. J. (2007). The experience of emotion. Annual Review of Psychology, 58, 373-403.

Barrett, L. F., \& Russell, J. A. (1999). The structure of current affect: Controversies and emerging consensus. Current Directions in Psychological Science, 8, 10-14.

Beck, A. T., Steer, R. A., \& Brown, G. K. (1996). Manual for the Beck Depression Inventory - II. San Antonio, TX: Psychological Corporation.

Becker, T. M., Cicero, D. C., \& Kerns, J. G. (2007, October). Disorganized speech, cognitive control deficits, and emotion traits in schizophrenia. Poster presented at the annual meeting of the Society for Research in Psychopathology, Iowa City, IA.

Berenbaum, H., Boden, M. T., Baker, J. P., Dizen, M., Thompson, R. J., \& Abramowitz, A. (2006). Emotional correlates of the different dimensions of schizotypal personality disorder. Journal of Abnormal Psychology, 115, 359-368.

Berenbaum, H., \& Connelly, J. (1993). The effects of stress on hedonic capacity. Journal of Abnormal Psychology, 102, 474-481.

Berenbaum, H., \& Irvin, S. (1996). Alexithymia, anger, and interpersonal behavior. Psychotherapy and Psychosomatics, 65, 203-208.

Berenbaum, H., Snowhite, R., \& Oltmanns, T. F. (1987). Anhedonia and emotional responses to affect evoking stimuli. Psychological Medicine, 17, 677-684.
Blanchard, J. J., \& Neale, J. M. (1992). Medication effects: Conceptual and methodological issues in schizophrenia research. Clinical Psychology Review, 12, 345-361.

Brown, L. H., Silvia, P. J., Myin-Germeys, I., \& Kwapil, T. R. (2007). When the need to belong goes wrong: The expression of social anhedonia and social anxiety in daily life. Psychological Science, 18, 778-782.

Burbridge, J. A., \& Barch, D. M. (2007). Anhedonia and the experience of emotion in individuals with schizophrenia. Journal of Abnormal Psychology, 116, 30-42.

Chapman, J. P., Chapman, L. J., \& Kwapil, T. R. (1995). Scales for the measurement of schizotypy. In A. Raine \& T. Lencz (Eds.), Schizotypal personality (pp. 79-106). New York: Cambridge University Press.

Chapman, L. J., \& Chapman, J. P. (1983). Infrequency scale. Unpublished test.

Chapman, L. J., Chapman, J. P., Kwapil, T. R., Eckblad, M., \& Zinser, M. C. (1994). Putatively psychosis-prone subjects 10 years later. Journal of Abnormal Psychology, 103, 171-183.

Chapman, L. J., Chapman, J. P., \& Raulin, M. L. (1976). Scales for physical and social anhedonia. Journal of Abnormal Psychology, 85, 374-382.

Chapman, L. J., Chapman, J. P., \& Raulin, M. L. (1978). Body-image aberration in schizophrenia. Journal of Abnormal Psychology, 87, 399-407.

Chmielewski, P. M., Fernandes, L. O., Yee, C. M., \& Miller, G. A. (1995). Ethnicity and gender in scales of psychosis proneness and mood disorders. Journal of Abnormal Psychology, 104, 464-470.

Cohen, A. S., \& Minor, K. S. (in press). Emotional experience in patients with schizophrenia revisited: Meta-analysis of laboratory studies. Schizophrenia Bulletin.

Collins, L. M., Blanchard, J. J., \& Biondo, K. M. (2005). Behavioral signs of schizoidia and schizotypy in social anhedonics. Schizophrenia Research, 78, 309-322.

Crandall, C. S., Preisler, J. J., \& Aussprung, J. (1992). Measuring life event stress in the lives of college students: The Undergraduate Stress Questionnaire. Journal of Behavioral Medicine, 15, 627-662.

Eckblad, M., \& Chapman, L. J. (1983). Magical ideation as an indicator of schizotypy. Journal of Consulting and Clinical Psychology, 51, 215-225.

Eckblad, M., Chapman, L. J., Chapman, J. P., \& Mishlove, M. (1982). Revised Social Anhedonia Scale. Unpublished test.

Edell, W. S. (1995). The psychometric measurement of schizotypy using the Wisconsin Scales of Psychosis-Proneness. In G. A. Miller (Ed.), The behavioral high-risk paradigm in psychopathology (pp. 1-46). New Work: Springer-Verlag.

Ferguson, M. L., \& Katkin, E. S. (1996). Visual perception, anhedonia, and emotion. Biological Psychology, 42, 131-145.

Fiorito, E. R., \& Simons, R. F. (1994). Emotional imagery and physical anhedonia. Psychophysiology, 31, 513-521.

Fitzgibbons, L., \& Simons, R. F. (1992). Affective response to color-slide stimuli in subjects with physical anhedonia: A three-systems analysis. Psychophysiology, 29, 613-620.

Frijda, N. H., \& Sundararajan, L. (2007). Emotion refinement: A theory inspired by Chinese poetics. Perspectives on Psychological Science, 2, 227-241.

Gard, D. E., Kring, A. M., Gard, M. G., Horan, W. P., \& Green, M. F. (2007). Anhedonia in schizophrenia: Distinctions between anticipatory and consummatory pleasure. Schizophrenia Research, 93, 253-260.

Gasper, K., \& Bramesfeld, K. D. (2006). Should I follow my feelings? How individual differences in following feelings influence affective well-being, experience, and responsiveness. Journal of Research in Personality, 40, 986-1014.

Germans, M. K., \& Kring, A. M. (2000). Hedonic deficit in anhedonia: Support for the role of approach motivation. Personality and Individual Differences, 28, 659-672.

Goldberg, L. R. (1999). A broad-bandwidth, public domain, personality inventory measuring the lower-level facets of several five-factor models. 
In I. Mervielde, I. Deary, F. De Fruyt, \& F. Ostendorf (Eds.), Personality psychology in Europe (Vol. 7, pp. 7-28). Tilburg, the Netherlands: Tilburg University Press.

Gooding, D. C., Davidson, R. J., Putnam, K. M., \& Tallent, K. A. (2002). Normative emotion-modulated startle response individuals at risk for schizophrenia-spectrum disorders. Schizophrenia Research, 57, 109-120.

Gooding, D. C., Tallent, K. A., \& Matts, C. W. (2005). Clinical status of at-risk individuals 5 years later: Further validation of the psychometric high-risk strategy. Journal of Abnormal Psychology, 114, 170-175.

Hafner, H., \& an der Heiden, W. (2003). Course and outcome of schizophrenia. In S. R. Hirsch \& D. R. Weinberger (Eds.), Schizophrenia (2nd ed., pp. 101-141). Malden, MA: Blackwell.

Heller, W. (1994). Neuropsychological mechanisms of individual differences in emotion, personality, and arousal. Neuropsychology, 7, 476-489.

Horan, W. P., \& Blanchard, J. J. (2003). Neurocognitive, social, and emotional dysfunction in deficit syndrome schizophrenia. Schizophrenia Research, 65, 125-137.

Horan, W. P., Blanchard, J. J., Gangstead, S. W., \& Kwapil, T. R. (2004). The psychometric detection of schizotypy: Do putative schizotypy indicators identify the same latent class? Journal of Abnormal Psychology, 113, 339-357.

Horan, W. P., Green, M. F., Kring, A. M., \& Nuechterlein, K. H. (2006). Does anhedonia in schizophrenia reflect faulty memory for subjectively experienced emotions? Journal of Abnormal Psychology, 115, 496-508.

Kahneman, D., Krueger, A. B., Schkade, D. A., Schwarz, N., \& Stone, A. A. (2004, December 3). A survey method for characterizing daily life experience: The day reconstruction method. Science, 306, 1776-1780.

Kerns, J. G. (2005). Positive schizotypy and emotion processing. Journal of Abnormal Psychology, 114, 392-401.

Kerns, J. G. (2006). Schizotypy facets, cognitive control, and emotion. Journal of Abnormal Psychology, 115, 418-427.

Kerns, J. G., \& Becker, T. M. (2008). Communication disturbances, working memory, and emotion in people with elevated disorganized schizotypy. Schizophrenia Research, 100, 172-180.

Kerns, J. G., \& Berenbaum, H. (2000). Aberrant semantic and affective processing in people at risk for psychosis. Journal of Abnormal Psychology, 109, 728-732.

Kirkpatrick, B., Fenton, W. S., Carpenter, W. T., Jr., \& Marder, S. R. (2006). The NIMH-MATRICS consensus statement on negative symptoms. Schizophrenia Bulletin, 32, 214-219.

Kring, A. M. (1999). Emotion in schizophrenia: Old mystery, new understanding. Current Directions in Psychological Science, 8, 160-163.

Kwapil, T. R. (1998). Social anhedonia as a predictor of the development of schizophrenia-spectrum disorders. Journal of Abnormal Psychology, 107, $558-565$.

Lambie, J. A., \& Marcel, A. J. (2002). Consciousness and the varieties of emotion experience: A theoretical framework. Psychological Review, 109, 219-259.

Lang, P. J., Bradley, M. M., \& Cuthbert, B. N. (2005). International Affective Picture System (IAPS): Affective ratings of pictures and instruction manual (Technical Report A-6). Gainesville, FL: University of Florida.

Larsen, R. J., Billings, D. W., \& Cutler, S. E. (1996). Affect intensity and individual differences in informational style. Journal of Personality, 64, 185-207.

LeDoux, J. E. (2000). Emotion circuits in the brain. Annual Review of Neuroscience, 23, 155-184.
Lenzenweger, M. F. (1999). Schizophrenia: Refining the phenotype, resolving endophenotypes. Behaviour Research and Therapy, 37, 281-295.

Lucas, R. E., \& Diener, E. (2001). Understanding extraverts' enjoyment of social situations: The importance of pleasantness. Journal of Personality and Social Psychology, 81, 343-356.

Luh, K. E., \& Gooding, D. C. (1999). Perceptual biases in psychosis-prone individuals. Journal of Abnormal Psychology, 108, 283-289.

Martin, E. A., \& Kerns, J. G. (2008a). Facets of schizotypy and emotional control. Manuscript in preparation.

Martin, E. A., \& Kerns, J. G. (2008b, September). Social anhedonia and increased emotional but not cognitive conflict. Poster presented at the Society for Research in Psychopathology, Pittsburgh, PA.

Martin, E. A., \& Kerns, J. G. (2008c). Social anhedonia associated with poor emotional but not poor cognitive control. Manuscript in preparation.

Mason, O. (1995). A confirmatory factor analysis of the structure of schizotypy. European Journal of Personality, 9, 271-281.

Meehl, P. E. (1962). Schizotaxia, schizotypy, schizophrenia. American Psychologist, 17, 827-838.

Miller, G. A., \& Chapman, J. P. (2001). Misunderstanding analysis of covariance. Journal of Abnormal Psychology, 110, 40-48.

Myin-Germeys, I., Delespaul, P. A. E. G., \& deVries, M. W. (2000). Schizophrenia patients are more emotionally active than is assumed based on their behavior. Schizophrenia Bulletin, 26, 847-854.

Phelps, E. A. (2006). Emotion and cognition: Insights from studies of the human amygdala. Annual Review of Psychology, 57, 27-53.

Preacher, K. J., Rucker, D. D., MacCallum, R. C., \& Nicewander, W. A. (2005). Use of the extreme groups approach: A critical reexamination and new recommendations. Psychological Methods, 10, 178-192.

Prince, J. D., \& Berenbaum, H. (1993). Alexithymia and hedonic capacity. Journal of Research in Personality, 27, 15-22.

Robinson, M. D., \& Clore, G. L. (2002). Belief and feeling: Evidence for an accessibility model of emotional self-report. Psychological Bulletin, 128, 934-960.

Rosenthal, R. (1991). Meta-analytic procedures for social research. Thousand Oaks, CA: Sage.

Ross, S. R., Lutz, C. J., \& Bailey, S. E. (2002). Positive and negative symptoms of schizotypy and the five-factor model: A domain and facet level analysis. Journal of Personality Assessment, 79, 53-72.

Schimmack, U., \& Diener, E. (1997). Affect intensity: Separating intensity and frequency in repeatedly measured affect. Journal of Personality and Social Psychology, 73, 1313-1329.

Shadish, W. R., Cook, T. D., \& Campbell, D. T. (2002). Experimental and quasi-experimental designs for generalized causal inference. New York: Houghton Mifflin.

Shrout, P. E., \& Fleiss, J. L. (1979). Intraclass correlations: Uses in assessing rater reliability. Psychological Bulletin, 86, 420-428.

VandenBos, G. R. (Ed.). (2006). APA dictionary of psychology. Washington, DC: American Psychological Association.

Watson, D., Gamez, W., \& Simms, L. J. (2005). Basic dimensions of temperament and their relation to anxiety and depression: A symptombased perspective. Journal of Research in Personality, 39, 46-66.

Received July 18, 2006

Revision received July 11, 2008

Accepted July 16, 2008 